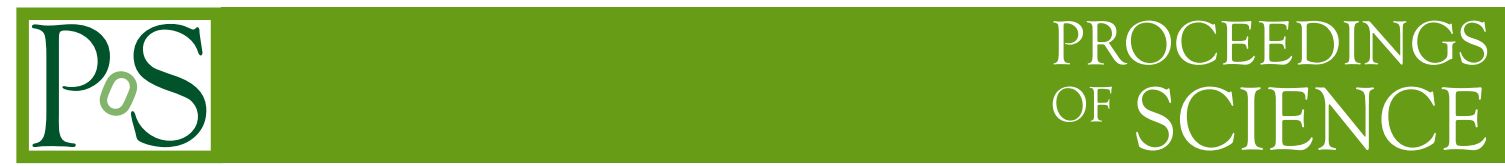

\title{
Search for new quarks with the ATLAS detector
}

\author{
G. Azuelos* on behalf of the ATLAS Collaboration \\ TRIUMF (Vancouver) and Université de Montréal, Canada \\ E-mail: georges.azueloseumontreal.ca
}

\begin{abstract}
Recent searches for vector-like quarks of the third generation using $3.2 \mathrm{fb}^{-1}$ of integrated luminosity with the ATLAS detector at the LHC at CERN are reported: Pair production searches are based on the presence of same-sign leptons, $b$-tagged jets and missing transverse momentum or on the requirement of multiple jets and $b$-tagged jets. The results are also sensitive to anomalous 4-top production by contact interaction or by an extra-dimensionsl model. Single production of heavy vector-like quarks is kinematically favoured, but is dependent on a sizable coupling to third generation standard model quarks. Limits on these couplings are reported.
\end{abstract}

38th International Conference on High Energy Physics

3-10 August 2016

Chicago, USA

${ }^{*}$ Speaker. 


\section{Introduction}

Vector-like quarks, for which both left and right chiralities are in the same representation of SU(2), are not excluded by the present measurements of the Higgs boon properties since, having no Yukawa coupling to the Higgs doublet, they do not contribute in loops to the gluon fusion production or decay of the Higgs boson and they do not cause vacuum instability in the SM [1,2]. They are generically predicted in many theories beyond the standard model (SM) such as Little Higgs models [3], composite Higgs models [4] or extra-dimension models [5]. They cancel the ultraviolet divergence in radiative corrections to the Higgs mass and they are often referred to as top partners since they are generally expected to couple mainly to the third generation quarks of the SM. Here, we report on recent results on the search for top partners in pair and single production with the ATLAS detector [6] at the LHC, from Run 2 with an integrated luminosity of $3.2 \mathrm{fb}^{-1}$.

Final states with the presence of b-jets and tops can give information on 4-top production, which has a low cross section in the SM of $\sim 9 \mathrm{fb}$, and on a possible 4-point interaction between top quarks in models of quark compositeness $[7,8,9]$. The contact interaction Lagrangian will be parametrized by $L_{4 t}=\left(C_{4 t} / \Lambda^{2}\right)\left(\bar{t}_{R} \gamma_{\mu} t_{R}\right)\left(\bar{t}_{R} \gamma_{\mu} t_{R}\right)$. These states can also be interpreted in terms of a model with two universal extra dimensions under real projective plane geometry (2UED/RPP) [10]. It is parameterized by the radii $R_{4}$ and $R_{5}$ with associated Kaluza-Klein states $m_{K K}=1 / R_{4}$ or $1 / R_{5}$. With Kaluza-Klein (KK) parity conservation, the model allows for a dark matter candidate. It predicts pair production of a KK photon $A(1,1)$ with mass $\sqrt{1+\xi^{2}} / R_{4}$, where $\xi=R_{4} / R_{5}$, that decay to $t \bar{t}$ with a branching fraction which will be assumed here to be $100 \%$.

\section{Events with b-jets and same-sign leptons}

Combined with the requirement of the presence of b-tagged jets and missing transverse momentum, a backgrround of SM events with same-sign leptons is very low, and good sensitivity can be obtained in the search for pair production of vector-like quarks: $T \bar{T}, B \bar{B}$, with $T \rightarrow Z t, H t$ or $B \rightarrow W t, Z b$ as well as $T_{5 / 3} \bar{T}_{5 / 3}$, with $T_{5 / 3} \rightarrow W t$. The analysis [11] can also search for evidence of SM production of 4 tops, or in particular for an excess of such events from contact interactions or from the 2UED/RPP model of extra dimensions.

$B, T$, and $T_{5 / 3}$ pair production is modelled using the Protos v2.2 [12] generator using the NNPDF2.3 [13] parton distribution functions (PDFs), with Pythia8 [14] used to model extra gluon emission and hadronization. Production of four top quarks is modelled under three scenarios: i) Standard Model, ii) contact interaction, and iii) 2UED/RPP. All three models are generated with MadGraph_aMC@NLO [15] at leading order, using the NNPDF PDFs followed by Pythia8. Backgrounds from $t \bar{t} W, t \bar{t} Z, t \bar{t} W W$ and $t \bar{t} H$ are simulated with MadGraph_aMC@NLO, while diboson and triboson backgrounds are simulated with Sherpa [16]. The dominant backgrounds, however, are detector-related: lepton charge misidentification and non-prompt and jets reconstructed as electrons. These are estimated respectively from $Z \rightarrow e e$ events, with a systematic uncertainty of 3-8\% and by a method which measures how "loosely" and "tightly" reconstructed leptons are related to true and false leptons in the data with a systematic uncertainty estimated at 13-26\% of the total background. 
Eight signal regions are defined, based on selection criteria on the scalar sum of the jet and lepton transerse momenta, $H_{T}$, on $E_{T}^{\text {miss }}$ and on the number of jets.

From these results, $95 \%$ limits are obtained on the mass of the $B, T$ and $T_{5 / 3}$ vector-like quarks, assuming branching ratios given by the model where the $B$ and $T$ quarks are isospin singlets: $m_{B}>830 \mathrm{GeV}, m_{T}>780 \mathrm{GeV}$ and $m_{T_{5 / 3}}>990 \mathrm{GeV}$. At high mass, the branching ratios to the third generation quarks tend to $50 \%$ for the charged current decay and $25 \%$ for each of the decays with a $\mathrm{Z}$ and with a Higgs boson, while the $T_{5 / 3}$ quark naturally decays $100 \%$ of the times to $W t$. Fig. 1 shows the contours of observed limits in the plane of $B R(B \rightarrow H b)$ versus $B R(B \rightarrow W t)$ and $B R(T \rightarrow H t)$ versus $B R(T \rightarrow W b)$. Limits on the 4-top cross section are: 95 (67) fb, assuming SM (contact) kinematics. Fig. 2 (left) shows the $95 \%$ limits on the coupling constant $\left|C_{4 t}\right|$ of the contact interaction model as a function of the scale of new physics and (right) the excluded region in the parameters of the 2UED/RPP model.
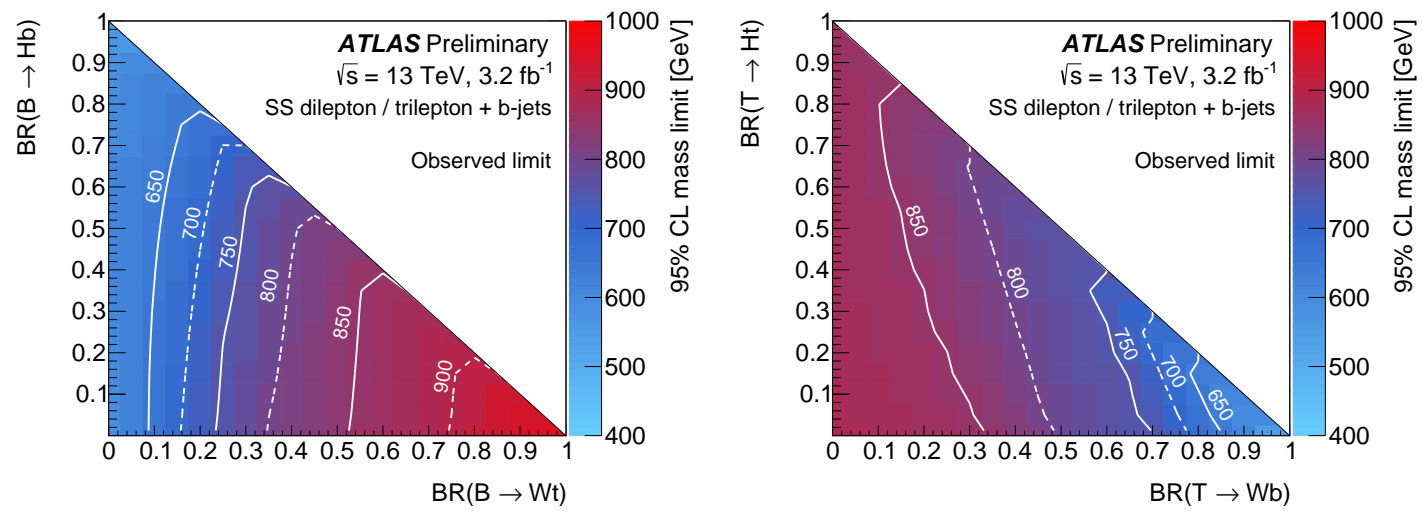

Figure 1: (a) Observed 95\% CL lower limit contours on the mass of (left) the B quark and (right) the T quark in the plane of branching ratios [11].
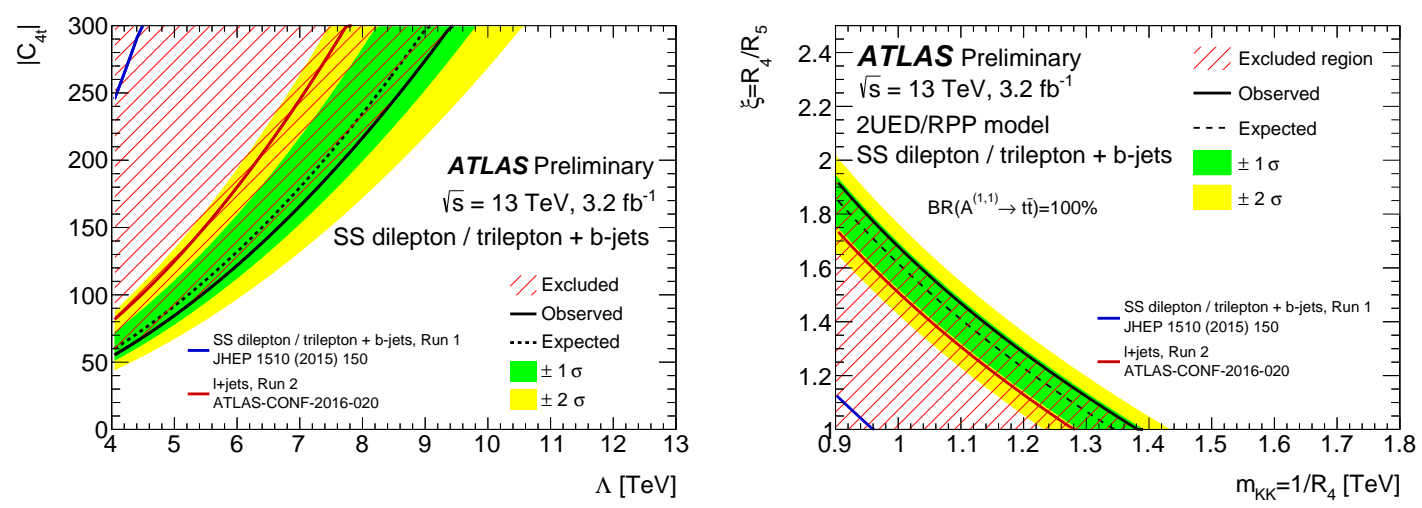

Figure 2: left: Expected and observed limits on the coupling constant $\left|C_{4 t}\right|$ in the contact interaction model obtained from the search for four-top-quark production as a function of the BSM physics energy scale $\Lambda$. The region in the upper left, corresponding to $\left|C_{4 t}\right| / \Lambda^{2}>3.5 \mathrm{TeV}^{-2}$, is excluded at 95\% CL. (c) Expected and observed limit in the $\left(m_{K K}=1 / R_{4}, \xi=R_{4} / R_{5}\right)$ plane for the $2 \mathrm{UED} / \mathrm{RPP}$ model [11]. 


\section{Events with leptons and jets}

The search for production of vector-like top quark pairs and of four top quarks was also carried out by selecting events with leptons and multiple jets in the final state [17]. After a preselection of events with one isolated lepton, different signal regions are defined based on the number of jets (6-8), at least two of which are b-tagged and with up to 2 jets required to be boosted top jets. The latter are reconstructed from large-radius $(\mathrm{R}=1.0)$ jets of mass $>300 \mathrm{GeV}$ made up of at least two subjets of smaller radius $(\mathrm{R}=0.4)$. The large- $\mathrm{R}$ jets are trimmed to remove subjets with $p_{T}<5 \%$ of the $p_{T}$ of the large-R jet. Fig 3 shows the jet multiplicity after the preselection and the large$\mathrm{R}$ jet mass distribution of events with at least 6 jets and $2 b$-tagged jets. Backgrounds $t \bar{t}, W t$ are simulated with PowhegBox[18] and MadGraph, $W+$ jets, dibosons by Sherpa and $t \bar{t}+W, Z$ or $H$ by MadGraph. The total systematic uncertainty on the background normalisation is approximately $24 \%$, with the largest contributions originating from uncertainties on $t \bar{t}+$ jets modelling, b-tagging, c-tagging and light-jet tagging efficiencies. The limits obtained on the mass of the T quarks are: $m_{T}$ (singlet) $>750 \mathrm{GeV}$ (or $900 \mathrm{GeV}$ if $B R(T \rightarrow W b)=1$. and $m_{T}$ (doublet) $>800 \mathrm{GeV}$.

This analysis [17], as well as one based based on a high multiplicity of jets (9-10) and at least $3 b$-tagged jets [19], yield limits on the production of 4-tops: $\sigma(4 t)<190(140)$ fb assuming SM (contact) interaction kinematics. Limits obtained on parameters of the contact interaction are: $\left|C_{4 t}\right| / \Lambda^{2}<4.8 \mathrm{TeV}^{-2}$ and of the 2ED/RPP model of extra dimensions: $1 / R_{4}>1.45 \mathrm{TeV}$.
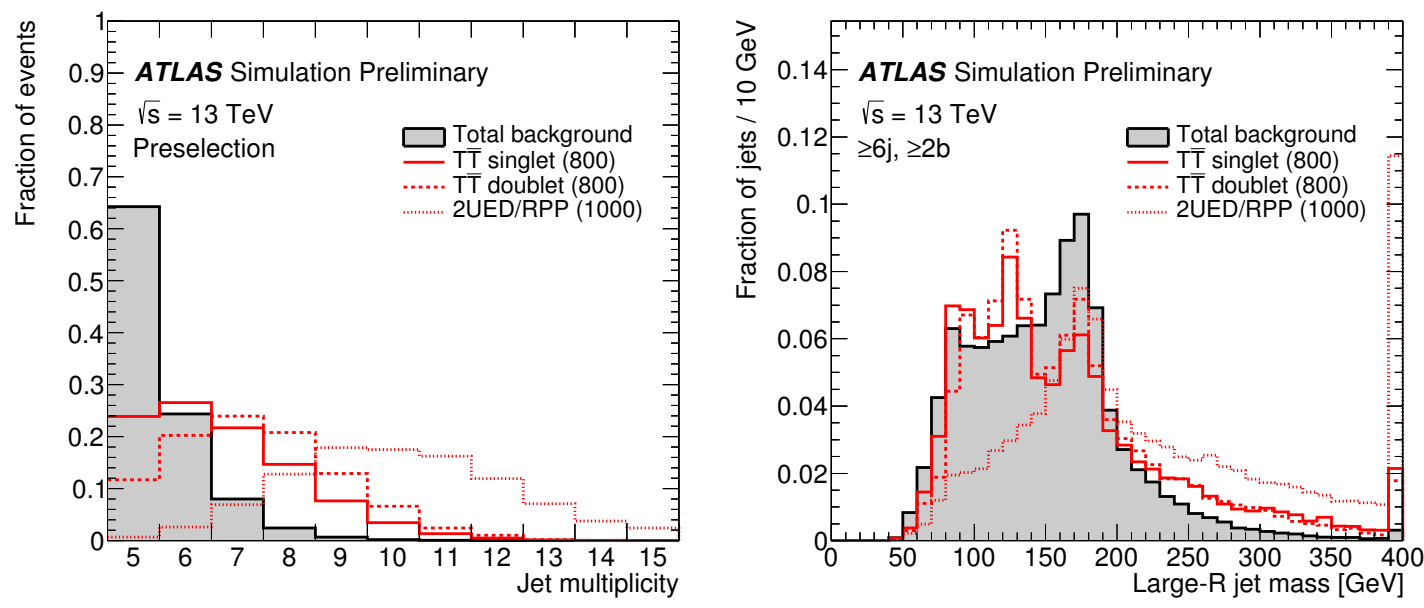

Figure 3: Left: jet multiplicity distribution after preselection of the total background (shaded histogram) and several signal scenarios considered in this search; Right: shape of the invariant mass distribution of selected large-R jets [17].

\section{Single Production: $T, Y_{4 / 3} \rightarrow W b$}

Single production of vector-like quarks, $q g \rightarrow q^{\prime} b+(W+b \rightarrow Q \rightarrow W b)$, is kinematically favored for high masses but depends on a sufficiently strong coupling to gauge bosons. This coupling 
can be expressed either as a mixing angle $\cos \theta_{L / R}$ of the SM $t$ quark with $T$ (and similarly for the $b-B$ mixing) [2] or more generally as a coupling, $c_{L / R}^{W b}$ in a phenomenological Lagrangian in an effective model [20], with the relation $c_{L / R}^{W b}=\sqrt{2} \sin \theta_{L / R}$.

In a recent analysis [21], simulated events for signal processes are generated at leading order (LO) with the Madgraph5_aMC@NLO generator using the NNPDF2.3 PDF set, and interfaced to Pythia8. The $T$ and $Y_{4 / 3}$ quarks have the same kinematic distributions, but the Y quark naturally decays $100 \%$ to $W b$. Background $t \bar{t}$ and single top are generated with Powheg-Box and Pythia, and $W$ or $Z+$ jets and dibosons with Sherpa. The event selection requires exactly one lepton, a high- $p_{T}$ $b$-tagged jet and a forward jet, a veto of a high $p_{T}$ central jet to suppress the $t \bar{t}$ background and a large opening angle between the lepton and leading jet. Control regions enriched in $W+$ jets and $t \bar{t}$ events are used to normalize the main backgrounds. Systematic uncertainties are dominated by uncertainties in $b$-tagging (10\%) and modelling of $W+$ jets at high $p_{T}(\sim 25 \%)$.

Assuming $\sqrt{\left(c_{L}^{W b}\right)^{2}+\left(c_{R}^{W b}\right)^{2}}=1 / \sqrt{2}$, a limit of $1.44 \mathrm{TeV}$ is obtained on the mass of the heavy quark $(B R=1.0)$. Fig. 4 shows limits obtained on the mixing angles $\sin \theta_{L}$ in the case of $T-t$ for the singlet case and $\sin \theta_{R}$ for $B-b$ mixing for the $(B, Y)$ doublet cases.
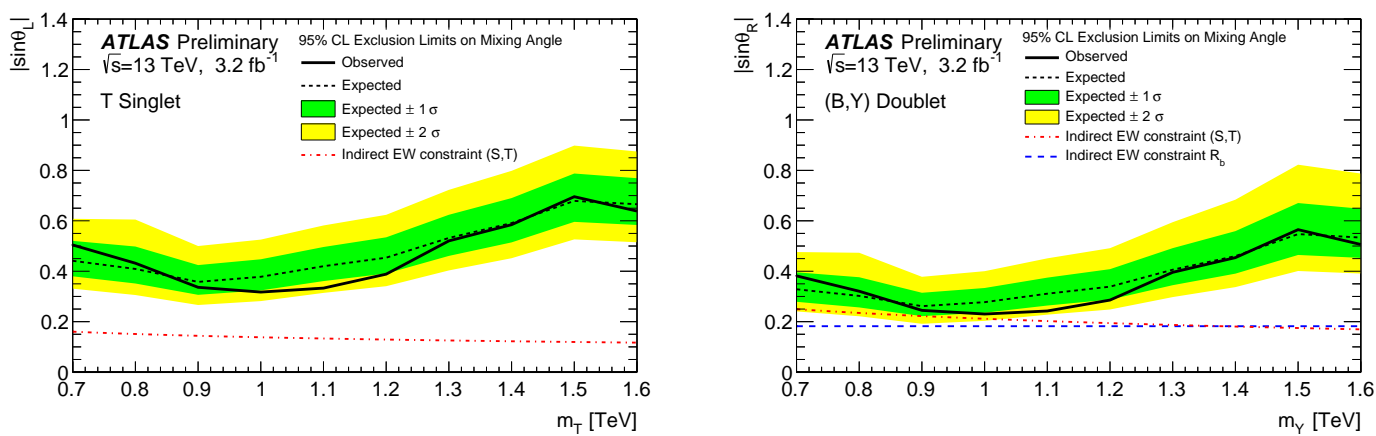

Figure 4: Limits at $95 \% \mathrm{CL}$ on (left) $\sin \theta_{L}$ for singlet T quark models assuming a $B R(T \rightarrow W b)=0.5$ and (right) on $\sin \theta_{R}$ for $(B, Y)$ doublet models as a function of the VLQ mass. As a comparison, constraints from electroweak precision observables taken from Ref. [2] are shown as well [21].

\section{Conclusion}

With early Run 2 data, based on $3.2 \mathrm{fb}^{-1}$ of integrated luminosity, improved limits have been obtained on masses of third generation vector-like quarks and four-top production cross section. Much more data is expected this year and in the next years and stronger limits, or hopefully a discovery, can be expected!

\section{References}

[1] PH Frampton et al., Quarks and leptons beyond the third generation, Phys Rep. 2000;330:263Đ348

[2] JA Aguilar-Saavedra et al., A handbook of vector-like quarks: mixing and single production, Phys.Rev. D88 (2013) 094010 
[3] M. Schmaltz and D. Tucker-Smith, Little Higgs Review, Ann. Rev. Nucl. Part. Sci. 55 (2005) 229

[4] R. Contino, L. Da Rold and A. Pomarol, Light custodians in natural composite Higgs models, Phys.Rev. D75 (2007) 055014

[5] T. Appelquist, H-C Cheng and BA Dobrescu, Bounds on Universal Extra Dimensions. Phys.Rev.D64:035002,2001

[6] ATLAS Collaboration, The ATLAS Experiment at the CERN Large Hadron Collider, JINST 3 (2008) S08003

[7] B. Lillie, J. Shu, and T. M. P. Tait, Top Compositeness at the Tevatron and LHC, JHEP 0804 (2008) 087

[8] A. Pomarol and J. Serra, Top quark compositeness: Feasibility and implications, Phys. Rev. D 78 (2008) 074026

[9] C. Degrande et al., Non-resonant new physics in top pair production at hadron colliders, JHEP 1103 (2011) 125

[10] G. Cacciapaglia et al., Heavy Vector-like quark with charge 5/3 at the LHC JHEP 1303 (2013) 004

[11] ATLAS Collaboration, Search for new physics using events with b-jets and a pair of same charge leptons in $3.2 \mathrm{fb}^{-1}$ of pp collisions at $\sqrt{\mathrm{s}}=13 \mathrm{TeV}$ with the ATLAS detector,

ATLAS-CONF-2016-032

[12] J. A. Aguilar-Saavedra, Identifying top partners at LHC, JHEP 0911 (2009) 030

[13] S. Carrazza, S. Forte, and J. Rojo, Parton Distributions and Event Generators, Proceedings, 43rd International Symposium on Multiparticle Dynamics (ISMD 13), 201389

[14] T. Sjostrand, S. Mrenna, and P. Z. Skands, A Brief Introduction to PYTHIA 8.1, Comput. Phys. Commun. 178 (2008) 852

[15] J. Alwall et al., The automated computation of tree-level and next-to-leading order differential cross sections, and their matching to parton shower simulations, JHEP 1407 (2014) 079

[16] T. Gleisberg et al., Event generation with SHERPA 1.1, JHEP 0902 (2009) 007

[17] ATLAS Collaboration, Search for production of vector-like top quark pairs and of four top quarks in the lepton-plus-jets final state in pp collisions at $\sqrt{s}=13 \mathrm{TeV}$ with the ATLAS detector", ATLAS-CONF-2016-013

[18] S. Alioli et al., A general framework for implementing NLO calculations in shower Monte Carlo programs: the POWHEG BOX, JHEP 1006 (2010) 043

[19] ATLAS Collaboration, Search for four-top-quark production in final states with one charged lepton and multiple jets using $3.2 \mathrm{fb}^{-1}$ of proton-proton collisions at $\sqrt{\mathrm{s}}=13 \mathrm{TeV}$ with the ATLAS detector at the LHC", ATLAS-CONF-2016-020

[20] O. Matsedonskyi, G. Panico and A. Wulzer, On the Interpretation of Top Partners Searches, JHEP 1412 (2014) 097

[21] ATLAS Collaboration, Search for single production of vector-like quarks decaying into Wb in pp collisions at $\sqrt{s}=13 \mathrm{TeV}$ with the ATLAS detector, ATLAS-CONF-2016-072 